\title{
Review of: "Disentangling the molecular mechanisms underlying the retrieval and extinction of morphine withdrawal-associated memories in the basolateral amygdala and dentate gyrus."
}

Potential competing interests: The author(s) declared that no potential competing interests exist.

I found this article of great interest for two reasons. The first reason is that withdrawal-associated memories are crucial for drug relapse, and the authors investigate the mechanisms underlying the retrieval and extinction of morphine withdrawal-associated memories in this article. The second reason is that this article showed that the changes of neuroplastic proteins level are regional-dependent. 